\title{
Calidad del servicio académico para el licenciamiento y la acreditación de las Universidades Peruanas
}

Quality of the academic service for the licensing and accreditation of Peruvian Universities Qualidade do serviço acadêmico para licenciamento e credenciamento de universidades peruanas

\section{ARTÍCULO GENERAL}

Pedro Francisco Vásquez Mora

p.vasquezm@utem.cl

https://orcid.org/0000-0001-7822-4643

Universidad Nacional Federico Villareal, Lima - Perú

\author{
Domingo Hernández Celis \\ dr.domingohernandezcelis@gmail.com \\ https://orcid.org/0000-0002-9759-4436
}

Universidad Nacional Federico Villareal, Lima - Perú

Recibido 07 de Abril 2021 | Arbitrado y aceptado 19 de Junio 2021 | Publicado en 03 Setiembre 2021

\section{RESUMEN}

El problema se ha identificado en el proceso de licenciamiento y la correspondiente acreditación de las instituciones indicadas. El licenciamiento es un proceso administrativo que consiste en la verificación, dispuesto por Ley, por parte de la Superintendencia Nacional de Educación Superior Universitaria (SUNEDU) de las condiciones básicas de calidad que deben reunir las Universidades Peruanas, tanto públicas como privadas. Esta problemática se expresa en la siguiente pregunta: ¿Cómo influye la calidad del servicio académico en el licenciamiento y acreditación de las Universidades Públicas? Por otra parte, ante la problemática se propone la solución a través de la formulación de la hipótesis: La calidad del servicio académico influye en grado estadísticamente significativo en el licenciamiento y acreditación de las Universidades Públicas. Dicha hipótesis ha quedado demostrada por el trabajo de campo realizado al respecto. Asimismo, este trabajo se ha orientado al siguiente objetivo: Determinar la influencia de la calidad del servicio académico en el licenciamiento y acreditación de las Universidades Públicas. En general se concluye que la calidad del servicio académico influye en grado estadísticamente razonable en el licenciamiento y acreditación de las Universidades Públicas, como privadas. Palabras clave: Calidad del servicio académico; licenciamiento institucional; acreditación institucional y Universidades Públicas.

\section{ABSTRACT}

The problem has been identified in the licensing process and the corresponding accreditation of the indicated institutions. Licensing is an administrative process that consists of the verification, provided by law, by the National Superintendency of Higher University Education (SUNEDU) of the basic quality conditions that Peruvian universities, both public and private, must meet. This problem is expressed in the following question: How does the quality of the academic service influence the licensing and accreditation of Public Universities? On the other hand, faced with the problem, the solution is proposed through the formulation of the hypothesis: The quality of the academic service has a statistically significant influence on the licensing and accreditation of Public Universities. This hypothesis has been demonstrated by the field work carried out in this regard. Likewise, this work has been oriented to the following objective: To determine the influence of the quality of the academic service in the licensing and accreditation of Public Universities. In general, it is concluded that the quality of the academic service has a statistically reasonable influence on the licensing and accreditation of Public and Private Universities.

Keywords: Quality of academic service; institutional licensing; Institutional accreditation and Public Universities.

\section{RESUMO}

O problema foi identificado no processo de licenciamento e correspondente credenciamento das instituições indicadas. O licenciamento é um processo administrativo que consiste na verificação, prevista em lei, pela Superintendência Nacional de Ensino Superior Universitário (SUNEDU), das condições básicas de qualidade que as universidades peruanas, públicas e privadas, devem atender. Esse problema se expressa na seguinte questão: Como a qualidade do serviço acadêmico influencia o licenciamento e o credenciamento das Universidades Públicas? Por outro lado, face ao problema, a solução é proposta através da formulação da hipótese: A qualidade do serviço académico tem uma influência estatisticamente significativa no licenciamento e acreditação das Universidades Públicas. Esta hipótese foi demonstrada pelo trabalho de campo realizado a este respeito. Da mesma forma, este trabalho orientou-se para o seguinte objetivo: Determinar a influência da qualidade do serviço académico no licenciamento e acreditação de Universidades Públicas. De uma forma geral, conclui-se que a qualidade do serviço académico tem uma influência estatisticamente razoável no licenciamento e acreditação de Universidades Públicas e Privadas.

Palavras-chave: Qualidade do serviço acadêmico; licenciamento institucional; Acreditação institucional e universidades públicas. 
Introducción

\section{Estado actual del problema: Al respecto se han identificado los siguientes síntomas:}

Gran parte de las universidades no cumplen los requisitos para el licenciamiento institucional que es un procedimiento legal que consiste en verificar las condiciones básicas de calidad en la educación superior universitaria. Actualmente es imprescindible para prestar el servicio educativo, las universidades peruanas deben obtener necesariamente la denominada licencia de funcionamiento institucional. Para mayores detales, el licenciamiento es un procedimiento técnico que consiste en verificar el cumplimiento de diversos mecanismos institucionales como el de inserción laboral, infraestructura adecuada, planes de investigación y objetivos académicos, entre otros aspectos establecidos en las normas legales respectivas; los mismos que a la fecha no son cumplidos por más del 70\% de las universidades del país. En otro marco, también se ha determinado la falta acreditación de facultades, escuelas y programas de las universidades tanto públicas como privadas. Al respecto es oportuno aclarar que la acreditación universitaria es un proceso, actualmente opcional, para contar con una certificación de calidad en los procesos internos de las universidades peruanas, lo cual está establecido en la Ley Universitaria. Para mayor detalle existen varios tipos de acreditación: Acreditación institucional, acreditación de carreras, acreditación de programas de postgrado y acreditación de especialidades del área de salud y otras. Los beneficios de la acreditación tanto institucional o de carreras y programas son estudiar en una entidad que cuenta con una certificación respecto de la implementación de sus mecanismos de aseguramiento de la calidad. Lo cual a la fecha no se cuenta e incluso ha sido abandonado por ser solo un aspecto opcional, porque hay exigencia total por el licenciamiento.

Los hechos o situaciones producidas por la existencia de los síntomas antes indicados son los siguientes: Se ha determinado la aplicación desarticulada de políticas, estrategias, procesos y procedimientos que no facilitan el licenciamiento, acreditación de las universidades peruanas; también se ha determinado la falta de seguimiento preciso y eficiente de la información necesaria que permita no dejar de lado nunca las perspectivas a mediano y largo plazo para saber si se están cumpliendo las metas, objetivos, misión y visión institucional. Por otro lado, las situaciones que podrían darse 
si se siguen presentando los síntomas antes indicados son las siguientes: Las universidades, algunas más que otras, no aprovecharían las oportunidades para licenciarse ni acreditarse institucionalmente como a nivel de carreras y/o programas académicos; tampoco aprovecharían las fortalezas y oportunidades que le permitan cumplir sus metas, objetivos que son elementos trascendentes. La situación problemática no permitiría la existencia de un ambiente propicio para el licenciamiento y la acreditación de las universidades peruanas; asimismo no podrán tener mejora continua, innovación y creatividad institucional.

Según el Portal Educación en Red (21 de marzo del 2018) Universidades tienen problemas económicos para obtener el licenciamiento, según información de la SUNEDU. Al respecto se tiene lo siguiente: La Universidad Nacional de Arequipa se encuentra en un plan de adecuación: El plazo para obtener el licenciamiento de las universidades vence en el 2019, pero las instituciones públicas tienen dificultades para lograr este permiso que les permite continuar con la prestación del servicio educativo. Por su parte Contreras (18 de setiembre del 2017) señala que, en casi dos años, solo tres universidades públicas han logrado su licenciamiento.

\section{Problema general: ¿Cómo influye la calidad del servicio académico en el} licenciamiento y acreditación de las Universidades Públicas?

Problemas específicos: 1) ¿Cómo influye la implementación de tecnología de la información y comunicación en el licenciamiento y acreditación de las Universidades Públicas? 2) ¿Cómo influye la capacitación certificación docente en el licenciamiento y acreditación de las Universidades Públicas? 3) ¿Cómo influye la didáctica universitaria eficiente en el licenciamiento y acreditación de las Universidades Públicas? 4) ¿Cómo influye el rendimiento académico adecuado en el licenciamiento y acreditación de las Universidades Públicas? 5) ¿Cómo influye la formación académica óptima en el licenciamiento y acreditación de las Universidades Públicas?

Antecedentes nacionales: Zárate (2016) señala que la calidad de los servicios estudiantiles para alumnos de maestría puede ser considerada actualmente como una ventaja competitiva en relación a otras universidades. Por su parte Vargas (2017) establece que la calidad de los servicios académicos y administrativos depende mucho del entendimiento y compromiso institucional. Entre tanto, para Aguirre y Rodríguez (2017) para mantener una organización en el mercado, es necesario entre otras cosas 
mejorar continuamente el lugar de trabajo, enfocándolo hacia la calidad de bienes y servicios, haciendo que esta actitud sea un factor que prevalezca en todas las actividades. En el mimo sentido para Tamashiro (2018) en su Tesis: Política de aseguramiento de la calidad en la educación superior y la administración estratégica, Universidad Norbert Wiener, año 2016; considera que fue necesario replantear y reorientar los procesos establecidos, a fin de cumplir con cada una de las especificaciones y exigencias señaladas por las instancias correspondientes.

Antecedentes internacionales: López (2016) indica que el concepto de calidad total o gestión de la calidad total es un concepto que ha cambiado su significado adaptándose a las demandas del mercado; la concepción de calidad ha surgido en un ámbito de producto y de empresa privada, más que en ámbito de los servicios y de las organizaciones en general. Para Araiza, Zambrano y Ramírez (2016) la UNESCO define calidad en la educación superior como un concepto multidimensional de múltiples niveles, dinámico, que se relaciona con los elementos contextuales de un modelo educacional, con la misión y fines institucionales y con estándares específicos dentro de un sistema, institución, programa o disciplina determinados. En cambio, para Vergara y Quesada (2011) consideran que la calidad en el servicio de un establecimiento educativo se ve reflejada en la conformidad y la satisfacción que experimenta la comunidad académica sobre los diferentes servicios prestados por éste. En cambio, Álvarez, Chaparro y Reyes (2017) considera que la gestión de la calidad en la educación superior promueve cambios positivos al interior de la universidad en cuatro componentes básicos: dirección y liderazgo, desarrollo de procesos académicos, desempeño de los equipos de trabajo y comportamiento de los actores individuales. Entre tanto, Campo (2017) dice que en la Educación Superior, en diferentes continentes y países, se han diseñado sistemas de calidad con definición de conceptos, determinación de variables, establecimiento de criterios de calidad, implementación de sistemas de control, aseguramiento y gestión de calidad; como también se han desarrollado modelos, instrumentos y procedimientos e indicadores de evaluación que permiten medir y evaluar el aseguramiento de la calidad.

\section{Bases teóricas:}

Calidad del servicio académico: Según Vargas y Aldana (2014) la calidad de servicio educativo es la búsqueda de lo mejor en provecho de los estudiantes. De similar 
manera Acuña (2014) considera que la calidad de servicio educativo es un conjunto de actividades ejecutadas por los diversos agentes a cargo de las funciones cuyos procesos siguen los procedimientos diseñados para lograr un servicio de calidad prefijado para cada uno de los productos que se ofrezcan. Se suma Seto (2014) el cual indica la calidad de servicio es considerada tanto por las empresas comerciales como las empresas de servicios, como arma estratégica para poder sobrevivir en el mercado y especialmente en entornos altamente competitivos. Anteriormente, Ishikawa \& Cárdenas (2013) ya decía que el alineamiento de los procesos (servicio al cliente), nos garantiza una sincronización total de la cadena de suministro y la cadena de valor para crear el valor definido por la estrategia y esperado por los clientes, proceso denominado alineamiento horizontal o peloteo. En cambio, para Lizcano (2014) en el ámbito de la administración la preocupación por la calidad no puede considerarse algo nuevo, sino que lo que ha variado es la orientación que se le ha dado a la misma a lo largo del tiempo, siendo posible distinguir tres grandes etapas: una primera fase, en la que se prima el respeto a las normas y los procedimientos; una segunda fase, en la que se asimila la calidad a la eficacia y una tercera fase, en la que se entiende la calidad como la satisfacción de las necesidades de los ciudadanos. Por su parte para Swiss (2012) los principales problemas que entraña la aplicación de la filosofía de gestión de la calidad total hacen referencia a las dificultades asociadas a la definición de los clientes de las entidades universitarias y a las derivadas de las características específicas de los servicios académicos. Para RuizOlalla (2012) considera que en lo que respecta a los conceptos de servicio y calidad del servicio, de acuerdo con el término servicio hace referencia a aquellas prestaciones que tienen como objetivo satisfacer las necesidades de los clientes.

Licenciamiento institucional: Según la Superintendencia Nacional de Educación Superior Universitaria-SUNEDU (10 de noviembre del 2017) el licenciamiento es un procedimiento que consiste en verificar condiciones básicas de calidad en la educación superior universitaria que reciben nuestros jóvenes.

Acreditación universitaria: Para Duarte (2014) con la promulgación de la Ley No 28740 (ley del SINEACE), se inicia el camino a la acreditación de la calidad de las instituciones educativas y de sus programas; siendo las universidades y sus carreras profesionales y programas de posgrado competencia del Consejo Nacional de Evaluación, Acreditación, Certificación de la Calidad de la Educación Universitaria CONEAU. Para Duarte (2014) la acreditación es el procedimiento que reconoce 
formalmente que la carrera profesional universitaria cumple con los estándares de calidad previamente establecido por él, como consecuencia del informe de evaluación satisfactorio presentado por la entidad evaluadora. Para Duarte (2014) el proceso de acreditación consta de las siguientes etapas: a) Etapa previa al proceso de Acreditación; b) Autoevaluación: Por su parte, Armendáriz (2014) el Sistema Nacional de Evaluación, Acreditación y Certificación de la Calidad Educativa -SINEACE, fue introducido en el marco legal peruano a través de los artículos 14, 15 y 16 de la Ley General de Educación - 28044, pero su regulación específica se ha dado a través de la Ley 28740 publicada el 23 de mayo de 2006. Esta última Ley establece los principios, finalidad y, funciones del Sistema, así como los aspectos generales de su organización interna.

Universidades peruanas: Según el Congreso de la República (10 de julio del 2014) la nueva Ley Universitaria tiene por objeto normar la creación, funcionamiento, supervisión y cierre de las universidades.

\section{Marco conceptual:}

Implementación de tecnología de la información y comunicación: Es imprescindible actualmente la implementación de la tecnología de la información y comunicación para gestionar de mejor manera las instituciones.

Capacitación y certificación docente: Los docentes de las universidades tienen que disponer de la capacitación suficiente y competente para llevar a cabo sus funciones académicas. La capacitación les asegura un buen servicio académico en beneficio de sus alumnos y para la entidad y para el licenciamiento y acreditación de las universidades.

Didáctica universitaria eficiente: La didáctica es el arte de enseñar, pero esta tiene que ser eficiente, en el sentido que tiene que hacerse utilizando ventajosamente todos los recursos como ocurre ahora con la aplicación de la tecnología de la información y comunicación con las herramientas cibernéticas.

Rendimiento académico adecuado: El rendimiento académico es el resultado del trabajo de los docentes y sus alumnos, es una suerte de sumatoria del trabajo de ambos. Un alto rendimiento trae ventajas para todos y especialmente para los discentes o alumnos de las instituciones académicas, también es un indicador del buen trabajo docente. 
Formación académica óptima: La calidad del servicio académico de las universidades conlleva la mejor formación académica de los egresados de dichas entidades y en ese sentido les traerá mejores oportunidades laborales tanto en forma dependiente como especialmente independiente, formando sus propias empresas para atender la demanda de bienes y servicios del mercado.

Infraestructura adecuada: La infraestructura adecuada es lo exige el proceso de licenciamiento entre otros indicadores de calidad, por tanto, las universidades tienen que invertir en dicha infraestructura, pero con sentido su suficiencia y competencia; es decir en la cantidad y calidad correspondientes y además adecuadamente ubicados para el acceso de los estudiantes, docentes y demás colaboradores.

Planes de investigación: Las universidades como entes económicos y sociales no pueden dejar de aplicar la planeación permanente de sus actividades y dentro de las mismas de los recursos que utilizan para prestar los servicios que requiere su comunidad educativa.

Inserción laboral: En las universidades debe existir una permanente preocupación porque sus egresados sean insertados en el mercado laboral formal y legal, lo cual será posible si dichas entidades disponen de calidad en los servicios académicos que prestan.

Autoevaluación para la acreditación: La autoevaluación es el examen realizado por las propias universidades como paso previo para lograr los estándares que permitirán alcanzar la acreditación correspondiente.

Evaluación externa para la acreditación: Además de la autoevaluación, resulta necesaria que un externo examine a la entidad para determinar si cumplen los parámetros para la acreditación correspondiente. Esto asegura más aun el cumplimiento de los procesos y procedimientos de acreditación y facilitará el cumplimi8ento de las normas que después serán verificadas por la entidad del estado correspondiente.

Justificación: La investigación beneficia a las universidades privadas; universidades públicas; comunidad universitaria y comunidad en general. A través de la investigación se aporta la teorización de la calidad de servicio académico y administrativo; licenciamiento institucional y la acreditación universitaria. Los beneficios que ofrece la investigación se da con la identificación de la problemática de las Universidades Peruanas y la solución de la problemática de las Universidades 
Peruanas. La investigación aporta un modelo de investigación que identifica la problemática y facilita la alternativa de solución para las Universidades Peruanas.

Objetivo general: Determinar la influencia de la calidad del servicio académico en el licenciamiento y acreditación de las Universidades Públicas.

Objetivos específicos: 1) Establecer la influencia de la implementación de tecnología de la información y comunicación en el licenciamiento y acreditación de las Universidades Públicas. 2) Determinar la influencia de la capacitación certificación docente en el licenciamiento y acreditación de las Universidades Públicas. 3) Establecer la influencia de la didáctica universitaria eficiente en el licenciamiento y acreditación de las Universidades Públicas. 4) Determinar la influencia del rendimiento académico adecuado en el licenciamiento y acreditación de las Universidades Públicas. 5) Establecer la influencia de la formación académica óptima en el licenciamiento y acreditación de las Universidades Públicas.

Hipótesis general: La calidad del servicio académico influye en grado estadísticamente significativo en el licenciamiento y acreditación de las Universidades Públicas.

Hipótesis específicas: 1) La implementación de tecnología de la información y comunicación influye en grado estadísticamente significativo en el licenciamiento y acreditación de las Universidades Públicas. 2) La capacitación certificación docente influye en grado estadísticamente significativo en el licenciamiento y acreditación de las Universidades Públicas. 3) La didáctica universitaria eficiente influye en grado estadísticamente significativo en el licenciamiento y acreditación de las Universidades Públicas. 4) El rendimiento académico adecuado influye en grado estadísticamente significativo en el licenciamiento y acreditación de las Universidades Públicas. 5) La formación académica óptima influye en grado estadísticamente significativo en el licenciamiento y acreditación de las Universidades Públicas.

\section{Materiales y métodos}

Tipo de investigación: Explicativa, descriptiva y correlacional.

Diseño de la investigación: El diseño fue no experimental. Al respecto, se observaron la calidad de servicio académico; y, el licenciamiento y acreditación de las 
universidades peruanas, tal y como se dieron en su contexto natural después de lo cual se obtuvo conclusiones.

Población: Este trabajo ha dado la oportunidad de trabajar con 10,200 personas relacionadas con la calidad de servicio académico; y, el licenciamiento y acreditación de las universidades peruanas.

Muestra: La muestra que se ha considerado fue de 370 personas, las cuales han tenido conocimientos razonables sobre los temas que se tratan en este trabajo.

Área de estudio: Calidad de servicio académico; licenciamiento institucional y acreditación de facultades de las universidades peruanas

Procedimientos estadísticos: Al respecto se ha aplicado el Chi cuadrado para determinar la asociación de las variables como el grado de significancia para rechazar la hipótesis nula en el marco de esta prueba estadística.

Aspectos éticos: Según Bunge (2014), en este marco todo lo que se expresa en este trabajo es verdad, por lo demás de aplica la objetividad e imparcialidad en el tratamiento de las opiniones y resultados; asimismo, no se da cuenta de aspectos confidenciales que manejan las Universidades Peruanas, públicas y privadas; grandes, medianas y pequeñas.

\section{Resultados}

El 90\% de los encuestados acepta que la calidad del servicio académico es la plataforma fundamental para alcanzar el licenciamiento y acreditación en las Universidades Peruanas. También el 90\% de los encuestados acepta que es necesaria la implementación de tecnología de información y comunicación para brindar un servicio académico de calidad en las Universidades Peruanas. El 88\% de los encuestados acepta que la tecnología de información y comunicación comprende ordenadores, programas informáticos y redes necesarias para convertirla, almacenarla, administrarla, transmitirla en las Universidades Peruanas. El 90\% de los encuestados acepta que la capacitación docente comprende las políticas y procedimientos planeados para preparar a potenciales profesores dentro de los ámbitos del conocimiento, actitudes, comportamientos y habilidades en las Universidades Peruanas. E1 88\% de los encuestados acepta que la 
certificación docente tiene como propósito que los docentes obtengan el Certificado en Competencias Docentes en las Universidades Peruanas.

Asimismo, se tiene que el $90 \%$ de los encuestados acepta que la didáctica universitaria comprende la mejor metodología de la enseñanza en las Universidades Peruanas. El 88\% de los encuestados acepta que la didáctica universitaria es un cuerpo teórico que estudia los problemas referentes a la enseñanza, con miras a posibilitar el aprendizaje de los estudiantes de las Universidades Peruanas. El 88\% de los encuestados acepta que el rendimiento académico adecuado comprende la evaluación del conocimiento adquirido por los estudiantes de las universidades peruanas. El 90\% de los encuestados acepta que el rendimiento académico adecuado es el producto de la asimilación del contenido de los programas de estudio, expresado en calificaciones dentro de una escala convencional en las Universidades Peruanas. El 88\% de los encuestados acepta que la formación académica óptima comprende la formación humanista de los estudiantes en las Universidades Peruanas

Por su parte, el $88 \%$ de los encuestados acepta que la formación académica óptima comprende la formación tecnológica y científica en las Universidades Peruanas. El 90\% de los encuestados acepta que el licenciamiento institucional es un procedimiento que consiste en verificar las condiciones básicas de calidad en la educación superior que reciben nuestros jóvenes en las Universidades Peruanas. El 90\% de los encuestados acepta que la acreditación es el reconocimiento público y temporal a la institución, área, programa o carrera profesional que voluntariamente ha participado en un proceso de evaluación de su gestión pedagógica, institucional y administrativa en las Universidades Peruanas. El 94\% de los encuestados acepta que la infraestructura adecuada comprende los locales, equipamiento y mobiliario para prestar los servicios académicos en las Universidades Peruanas. El 78\% de los encuestados acepta que modernamente no se puede concebir la falta de planes de investigación en las Universidades Peruanas. E1 94\% de los encuestados acepta que los planes de investigación facilitan el licenciamiento y también la acreditación en las Universidades Peruanas. El 94\% de los encuestados acepta que es un objetivo principal tanto del licenciamiento como de la acreditación lograr los mayores indicadores de inserción laboral de los egresados de las Universidades Peruanas. 
En cambio, el 94\% de los encuestados acepta que la inserción laboral cierra el proceso académico de las Universidades Peruanas. El 94\% de los encuestados acepta que la autoevaluación es la fase básica para el proceso de la acreditación en las universidades peruanas. El 89\% de los encuestados acepta que la autoevaluación permite identificar las fortalezas, las debilidades, oportunidades y amenazas en las Universidades Peruanas. El 94\% de los encuestados acepta que la evaluación externa para la acreditación en las Universidades Peruanas garantiza independencia para dicha evaluación. El 94\% de los encuestados acepta que la evaluación externa para la acreditación en las Universidades Peruanas garantiza objetividad para dicha evaluación.

Contrastación de la hipótesis: Respecto de la hipótesis general se tiene que la regla de decisión fue rechazar la hipótesis nula si el valor de $\mathrm{X}^{2}$ es mayor o igual al 5.00\%; al aplicar la estadística de prueba se tiene: X2 $=((78-82) 2) / 82)=0.1951=19.51 \%$ Por lo que siendo $19.51 \%>5.00 \%$, se rechaza la hipótesis nula y se acepta la hipótesis alternativa y se concluye que la calidad del servicio académico influye en grado estadísticamente significativo en el licenciamiento y acreditación de las Universidades Públicas. En lo referido a la hipótesis específica 1 se tiene que la regla de decisión fue rechazar hipótesis nula si el valor de $\mathrm{X}^{2}$ es mayor o igual al 5.00\%; al aplicar la estadística de prueba se tiene: $\mathrm{X} 2=((333-350) 2) / 350)=0.8257=82.57 \%$; entonces dado que $82.57 \%>5.00 \%$, se rechaza la hipótesis nula y se acepta la hipótesis alternativa y se concluye que la implementación de tecnología de la información y comunicación influye en grado estadísticamente significativo en el licenciamiento y acreditación de las Universidades Públicas. En relación a la hipótesis específica 2, la regla de decisión fue rechazar hipótesis nula si el valor de $X^{2}$ es mayor o igual a $5.00 \%$; luego al aplicar la estadística de prueba se tiene: $\mathrm{X} 2=((340-350) 2) / 350)=0.2857=$ $28.57 \%$; por lo que dado que $28.57 \%>5.00 \%$, se rechaza la hipótesis nula y se acepta la hipótesis alternativa; y se concluye que la capacitación certificación docente influye en grado estadísticamente significativo en el licenciamiento y acreditación de las Universidades Públicas.

En cuanto a la hipótesis específica 3, la regla de decisión fue rechazar hipótesis nula si el valor de $\mathrm{X}^{2}$ es mayor o igual a 5.00\%; entonces al aplicar la estadística de prueba se tiene: $\mathrm{X} 2=((350-360) 2) / 360)=0.2778=27.78 \%$ y dado que $27.70 \%>$ $5.00 \%$, se rechaza la hipótesis nula y se acepta la hipótesis alternativa y se concluye que 11 didáctica universitaria eficiente influye en grado estadísticamente significativo en el 
licenciamiento y acreditación de las Universidades Públicas. Luego, en cuanto a la hipótesis específica 4, la regla de decisión fue rechazar hipótesis nula si el valor de $\mathrm{X}^{2}$ es mayor o igual a 5.00\%; luego al aplicar la estadística de prueba se tiene: $\mathrm{X} 2=((340-$ $350) 2$ ) $/ 350)=0.2857=28.57 \%$; por lo que dado que $28.57 \%>5.00 \%$, se rechaza la hipótesis nula y se acepta la hipótesis alternativa y se concluye que el rendimiento académico adecuado influye en grado estadísticamente significativo en el licenciamiento y acreditación de las Universidades Públicas. En cuanto a la hipótesis específica 5, la regla de decisión fue rechazar hipótesis nula si el valor de $\mathrm{X}^{2}$ es mayor o igual a 5.00\%; luego al aplicar la estadística de prueba se tiene: $\mathrm{X} 2=((340-350) 2) / 350)$ $=0.2857=28.57 \%$; entonces, dado que $28.57 \%>5.00 \%$, se rechaza la hipótesis nula y se acepta la hipótesis alternativa y se concluye que la formación académica óptima influye en grado estadísticamente significativo en el licenciamiento y acreditación de las Universidades Públicas.

\section{Discusión}

El 90\% de los encuestados acepta que la calidad del servicio académico es la plataforma fundamental para alcanzar el licenciamiento y acreditación en las Universidades Peruanas. Este resultado es similar al 92\% presentado, aunque en otra dimensión espacial y temporal, por Araiza, M., Zambrano, A. y Ramírez, J. (2016). Calidad de los servicios universitarios: Una percepción desde sus actores en una escuela de negocios. (Tesis doctoral). Universidad Autónoma de Nuevo León. México. Dichos resultados son altos, por lo que apoyan el modelo de investigación desarrollado.

El 90\% de los encuestados acepta que el licenciamiento institucional es un procedimiento que consiste en verificar las condiciones básicas de calidad en la educación superior que reciben nuestros jóvenes en las Universidades Peruanas. Este resultado es similar al 93\% presentado, aunque en otra dimensión espacial y temporal, por Tamashiro, J. (2018). Política de aseguramiento de la calidad en la educación superior y la administración estratégica, Universidad Norbert Wiener, año 2016. (Tesis doctoral). Universidad Privada Norbert Wiener. Lima. Los resultados obtenidos denotan un alto resultado por lo que se deduce que apoyan a la investigación desarrollada. 


\section{Conclusiones}

Se ha determinado la influencia significativa de la calidad del servicio académico en el licenciamiento y acreditación de las Universidades Públicas. También se ha establecido la influencia significativa de la implementación de tecnología de la información y comunicación en el licenciamiento y acreditación de las Universidades Públicas. Luego se ha determinado la influencia significativa de la capacitación certificación docente en el licenciamiento y acreditación de las Universidades Públicas. Asimismo, se ha establecido la influencia significativa de la didáctica universitaria eficiente en el licenciamiento y acreditación de las Universidades Públicas. De la misma manera se ha determinado la influencia significativa del rendimiento académico adecuado en el licenciamiento y acreditación de las Universidades Públicas. Y también se ha establecido la influencia significativa de la formación académica óptima en el licenciamiento y acreditación de las Universidades Públicas.

\section{Recomendaciones}

Se recomienda a las autoridades y funcionarios tener en cuenta que la calidad del servicio académico influye en grado estadísticamente significativo en el licenciamiento y acreditación de las Universidades Públicas. También se recomienda a las autoridades y funcionarios tener en cuenta que la implementación de tecnología de la información y comunicación influye en grado estadísticamente significativo en el licenciamiento y acreditación de las Universidades Públicas. Asimismo, se recomienda a las autoridades y funcionarios tener en cuenta que la capacitación certificación docente influye en grado estadísticamente significativo en el licenciamiento y acreditación de las Universidades Públicas. Sin lugar a dudas, también se recomienda a las autoridades y funcionarios tener en cuenta que la didáctica universitaria eficiente influye en grado estadísticamente significativo en el licenciamiento y acreditación de las Universidades Públicas. También se recomienda a las autoridades y funcionarios tener en cuenta que el rendimiento académico adecuado influye en grado estadísticamente significativo en el licenciamiento y acreditación de las Universidades Públicas. Y finalmente se recomienda a las autoridades y funcionarios tener en cuenta que la formación académica óptima influye en grado estadísticamente significativo en el licenciamiento y acreditación de las Universidades Públicas. 


\section{Referencias}

Acuña, J. (2014), Mejoramiento de la calidad: un enfoque a los servicios. San José: Editorial Tecnológica de Costa Rica.

Aguirre, W. y Rodríguez, E. (2017). Evaluación de la calidad de servicio universitario desde la percepción de estudiantes y docentes en la Universidad Privada de la Selva Peruana, Iquitos 2017. (Tesis de pregrado). Universidad Privada De La Selva Peruana. Iquitos, Perú. Recuperado de:

http://repositorio.ups.edu.pe/bitstream/handle/ups/28/aguirre-espinozawildher.pdf? sequence $=4 \&$ isallowed $=y$

Álvarez, J., Chaparro, E. y Reyes, D. (2017). Estudio de la Satisfacción de los Estudiantes con los Servicios Educativos brindados por Instituciones de Educación Superior del Valle de Toluca. (Tesis doctoral). Universidad Autónoma del Estado de México. Recuperada de:

https://repositorio.uam.es/bitstream/handle/10486/668275/reice 13 2_1.pdf? sequ $\underline{\text { ence }=1}$

Araiza, M., Zambrano, A. y Ramírez, J. (2016). Calidad de los servicios universitarios: Una percepción desde sus actores en una escuela de negocios. (Tesis doctoral). Universidad Autónoma de Nuevo León. México. Recuperada de: https://www.researchgate.net/publication/316554022_calidad de los_servicios u niversitarios_una_percepcion_desde_sus_actores_en_una_escuela_de_negocios_q uality of services university a perception from their_actors in a business sc $\underline{\text { hool }}$

Armendáriz, G. (2014). El Sistema Nacional de Evaluación, Acreditación y Certificación de la Calidad Educativa-SINEACE y la calidad educativa. Lima: PUCP.

Bunge, M. (2014), La investigación científica. Su estrategia y su filosofia, Barcelona, Ariel.

Campo, M. (2017). Estudio comparativo de dos modelos de acreditación universitaria: la aplicabilidad de estándares y criterios a la Universidad Mayor de Chile. (Tesis doctoral). Universidad Nacional de Educación a Distancia. Madrid. Recuperada 
de: http://e-spacio.uned.es/fez/eserv/tesisuned:ed-pg-educacmscampo/campo_herrera_msoledad tesis.pdf

Congreso de la República (10 de julio del 2014). Ley No. 30220, nueva Ley

Universitaria. Ministerio de Educción. Lima. Recuperado de:

http://www.minedu.gob.pe/reforma-universitaria/pdf/ley_universitaria.pdf

Contreras, C. (18 de setiembre del 2017). En casi dos años, solo tres universidades públicas han logrado su licenciamiento. Diario La República. Lima. Recuperado dehttps://larepublica.pe/sociedad/1099835-en-casi-dos-anos-solo-tresuniversidades-publicas-han-logrado-su-licenciamiento

Duarte, L. (2014). Acreditación universitaria. Lima. Editorial San Marcos

Ishikawa, K. y Cárdenas, M. (2013). Calidad total. Bogotá Editorial Norma.

Lizcano, J. (2014). La mejora de las entidades en la Europa del Euro. Papel del sistema ABC/ABM. Madrid. Días de Santos Editorial.

López, A. (2016). La evaluación de la calidad en la Universidad Española. (Trabajo de investigación profesional). Universidad de Málaga. Málaga, España. Recuperado de: http://www.biblioteca.uma.es/bbldoc/tesisuma/16283211.pdf

Portal Educación en Red (21 de marzo del 2018). Universidades tienen problemas económicos para obtener el licenciamiento, según información de la SUNEDU. Lima. Recuperado de: https://noticia.educacionenred.pe/2018/03/universidadestienen-problemas-economicos-obtener-licenciamiento-segun-informacion146175.html

Ruiz-Olalla, A. (2012). Gestión de la calidad del servicio a través de indicadores externos. Monografías AECA, Asociación Española de Contabilidad y Administración de Empresas, Madrid.

Seto, D. (2014). De la calidad de servicio a la fidelidad del cliente. Madrid. ESIC Editorial.

Sistema Nacional de Evaluación, Acreditación y Certificación de la Calidad Educativa SINEACE (15 de enero 2018). Glosario de términos básicos de evaluación, 
acreditación, y certificación del SINEACE. Lima. Recuperado de:

Thttp://acreditacion.unsm.edu.pe/lib/docs/documentos_sineace_coneau/574_glosa rio\%20del\%20sineace $\% 20$-oct $\% 202010$ - [1].pdf

Superintendencia Nacional de Educación Superior Universitaria-SUNEDU (10 de noviembre del 2017). Licenciamiento institucional. Recuperado de: https://www.sunedu.gob.pe/direccion-de-licenciamiento/

Swiss, J. (2012) Adapting total quality management (TQM) to EGovernment, Public Administration Review.

Tamashiro, J. (2018). Política de aseguramiento de la calidad en la educación superior y la administración estratégica, Universidad Norbert Wiener, año 2016. (Tesis doctoral). Universidad Privada Norbert Wiener. Lima. http://repositorio.uwiener.edu.pe/bitstream/handle/123456789/1732/maestro\%20$\% 20$ tamashiro $\% 20$ tamashiro $\% 2 \mathrm{c} \% 20$ jaime.pdf? sequence $=1 \&$ isallowed=y

Vargas, J. (2017). Calidad de los Servicios Académicos y Administrativos de la Facultad de Ciencias Sociales de la Universidad Nacional de San Antonio Abad del Cusco, Según la Percepción del Personal docente y estudiantes, Año 2015. (Tesis de maestría). Universidad Católica de Santa María. Arequipa, Perú. Recuperado de: https://tesis.ucsm.edu.pe/repositorio/handle/ucsm/6473

Vargas, M. y Aldana de Vega, L. (2014). Calidad y Servicio. Conceptos y herramientas. Bogotá, D.C.: Ecoe Ediciones: Universidad de la Sabana.

Vergara, J. y Quesada, V. (2011). Análisis de la calidad en el servicio y satisfacción de los estudiantes de Ciencias Económicas de la Universidad de Cartagena mediante un modelo de ecuaciones estructurales. Revista Electrónica de Investigación Educativa, 13(1), 108-122. Recuperada de: http://redie.uabc.mx/vol13nol/contenido-vergaraquesada.html

Zárate, J. (2016). Calidad de servicios para estudiantes de posgrado de la Pontificia Universidad Católica del Perú. (Tesis de maestría). Pontificia Universidad Católica del Perú. Lima. Recuperado de: http://tesis.pucp.edu.pe/repositorio/bitstream/handle/123456789/7949/zarate_cord ova_jennifer_calidad_de_servicio.pdf? sequence $=1 \&$ isallowed $=\mathrm{y}$ 